\title{
OTRAS OPCIONES DE POLÍTICA MONETARIA. UN ANÁlISIS JURÍDICO DE LAS POLÍTICAS MONETARIAS NO CONVENCIONALES Y SU POSIBLE IMPLEMENTACIÓN EN LA REPÚBliCa DOMINICANA
}

\author{
Francisco A. Mota Pacheco ${ }^{a}$
}

Recibido: 9/12/2019・ Aprobado: 23/11/2020

\begin{abstract}
Cómo citar: Mota Pacheco, F. A. (2021). Otras opciones de política monetaria. Un análisis jurídico de las políticas monetarias no convencionales y su posible implementación en la República Dominicana. Ciencia, Economía y Negocios, 5(1), 5-35. Doi: https://doi.org/10.22206/ceyn.2021.v5i1.pp5-35
\end{abstract}

\section{Resumen}

Este ensayo presenta un análisis jurídico de las politicas monetarias no convencionales aplicadas en respuesta a la crisis financiera de 2008 y la actual crisis económica generada por el Covid-19 en el sistema jurídico monetario de la República Dominicana. Discutimos sobre la implementación de nuevas herramientas para ejecutar la politica monetaria, ya que los instrumentos convencionales en situaciones de crisis severas tienden a ser ineficaces. Abordamos los aspectos generales de la politica monetaria no convencional: naturaleza y fundamentos; asimismo, examinamos la aplicación de los diferentes instrumentos de politica monetaria no convencional por parte de los bancos centrales de las principales economías mundiales en el marco de la crisis financiera de 2008, asi como su excelente aplicación en la crisis económica actual. Finalmente, el documento demuestra cómo es posible su implementación en el sistema jurídico dominicano, conforme a la Ley Monetaria y Financiera 183-02, de fecha 3 de noviembre de 2002.

Palabras clave: regulación; política monetaria; políticas no convencionales; crisis financiera 2008; Gran Recesión;

Códigos del JEL: E52, E58, G18

a Jorge Luis Polanco, firma de abogados, Santo Domingo, República Dominicana. Correo-e: fmota@jorgeluispolanco.do

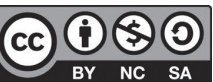




\title{
Other monetary policy options. A legal analysis of UNCONVENTIONAL MONETARY POLICIES AND THEIR POSSIBLE IMPLEMENTATION IN THE DoMinican RepUblic
}

\author{
Francisco A. Mota Pacheco ${ }^{a}$
}

Received: 12/9/2019 • Approved: 11/23/2020

\begin{abstract}
This essay presents a legal analysis of the unconventional monetary policies applied in response to the 2008 Financial Crisis and the current economic crisis generated by Covid-19 in the monetary legal system of the Dominican Republic. We argue about the implementation of new tools for executing monetary policy since conventional instruments in situations of severe crisis tend to be ineffective. We address the general aspects of unconventional monetary policy: nature and fundamentals; Likewise, we examine the application of the different instruments of unconventional monetary policy by the central banks of the leading world economies in the framework of the 2008 financial crisis, as well as their excellent application in the current economic crisis. Finally, the paper demonstrates how its implementation is possible in the Dominican legal system, following Monetary and Financial Law 183-02, dated November 3, 2002.
\end{abstract}

Keywords: regulation; monetary politics; unconventional policies; financial crisis 2008; Great Recession.

JEL codes: E52, E58, G18

a Jorge Luis Polanco, firma de abogados, Santo Domingo, Dominican Republic. E-mail: fmota@jorgeluispolanco.do 


\section{Introducción}

Una de las conclusiones de mi estudio de la Gran Depresión es que nosotros tenemos la tendencia a considerar que la ortodoxia es una estrategia segura. Pero la estrategia debe depender de la situación. En periodo de crisis, la ortodoxia puede resultar ser una muy mala estrategia. (Financial Times [FT], 2009)

Ben Bernanke

La política monetaria hace alusión al conjunto de actuaciones que emplea la autoridad monetaria para influir en la oferta, disponibilidad y costo de la moneda para cumplir su objetivo monetario, que puede ser la estabilidad de precios, el crecimiento económico sostenido o el aumento del empleo (Adelina, 2011).

Para cumplir con dichos fines, la autoridad encargada de ejecutar la política monetaria, que usualmente es un banco central (Fondo Monetario Internacional [FMI], 2018) se auxilia de instrumentos como: las tasas de interés, las operaciones de mercado abierto, el prestamista de última instancia, entre otros. En efecto, con la puesta en ejecución de los referidos instrumentos, los bancos centrales buscan mantener la tasa de interés en un nivel que resulte coherente con su objetivo monetario (Drumetz, Pfister y Sahuc, 2015).

Por su parte, se le denomina políticas monetarias no convencionales al conjunto de medidas excepcionales no reguladas empleadas por los bancos centrales de las economías más importantes del mundo, a través de sus instituciones reguladoras, como son la Reserva Federal de los Estados Unidos de Norteamérica (EE. UU.) (en adelante, "Fed"), el Banco de Inglaterra, el Banco Central Europeo (en lo adelante, "BCE") y, en cierta medida, el Banco Central de Japón, para detener los efectos de la Crisis Financiera Global del año dos mil ocho (2008) (Blanchard, Dell y Mauro, 2013).

Las medidas convencionales, por su parte, son aquellas que tradicionalmente se aplicaron antes de la crisis de 2008 como único medio para ejecutar de manera efectiva el objetivo de la política monetaria. Sin embargo, después de la crisis de 2008, estas medidas presentaron fallas, es decir, fueron insuficientes para cumplir con el fin de la política monetaria que en algunos casos es la estabilidad de precios, mientras que en otros es el crecimiento económico sostenido. 
El contexto histórico de esta investigación se sitúa en la crisis financiera de 2008, cuyos efectos impulsaron a muchos bancos centrales a disminuir su tipo de referencia hasta llegar a cero o negativo. Sin embargo, la situación se agravó con el colapso de los mercados monetarios, es decir, con la quiebra de importantes Entidades de Intermediación Financiera (en adelante, "EIF"), agencias aseguradoras, fondos de inversión, etc.

Lo indicado anteriormente trajo como consecuencia el agotamiento de los instrumentos convencionales de política monetaria, pues estos no podían impactar la economía de manera tal que se aumentara la liquidez y el crédito (Samuelson y Nordhaus, 2015). Es aquí donde nacen los instrumentos no convencionales de política monetaria, como respuesta a la insuficiencia de la política monetaria convencional.

Hoy, trece años después, este tema amerita ser examinado de cara a la crisis económica que el Fondo Monetario Internacional ha denominado "El Gran Confinamiento", que se ha generado por causa del Covid-19. Esta crisis, según las autoridades internacionales, apunta a ser una de las más fuertes, inclusive comparable a la Gran Depresión de 1929. Se diferencia de otras crisis económicas, porque el Covid-19 además de tener un alcance global, es un virus que solo puede combatirse con agresivas medidas de distanciamiento social como el confinamiento de la sociedad: esto implica la paralización de las actividades económicas y, por tanto, el declive económico.

El "Gran Confinamiento" ha obligado a los gobiernos a implementar una serie de medidas económicas para contrarrestar los efectos de la crisis en un contexto de grandes turbulencias e incertidumbres. Dentro de esas medidas económicas, debemos hacer énfasis en el paquete de políticas monetarias que se ha estado aplicando: esto incluye tanto políticas monetarias convencionales como no convencionales; sin embargo, nuestro enfoque será en las no convencionales.

Resulta de capital importancia estudiar estas políticas monetarias no convencionales, debido a que, en la actual crisis económica, al igual que en la crisis financiera de 2008, las autoridades monetarias tienen una tendencia a bajar los tipos de interés de referencia como instrumento monetario para dinamizar la economía. La caída brusca de los mercados monetarios ha acentuado la necesidad de aplicar esas medidas especiales 
de flexibilización monetaria para propiciar la liquidez y el mantenimiento del crédito.

Esta investigación abarca aspectos económicos y jurídicos: se refiere a aspectos económicos en tanto estudiamos las instituciones básicas de la política monetaria que nos permite entender el objeto de la regulación que se pretende hacer, usando las herramientas jurídicas apropiadas; es jurídica también, porque abordamos el tema desde la óptica de la regulación monetaria, que es importante para la estabilidad económica de cualquier país. Esto así en razón de que, si la autoridad monetaria facultada para garantizar el valor de la moneda no actúa apegada a efectivos controles jurídicos, nuestro desarrollo no estaría garantizado.

Las crisis tienen efectos devastadores y pueden impactar las políticas monetarias convencionales, las cuales podrían incluso agotarse, dejando a la autoridad monetaria sin herramientas efectivas, como lo ocurrido en la crisis financiera de 2008. De ahí nace el interés en abordar este tema, con el fin de poder brindar una aproximación teórico-jurídica sobre la posibilidad de usar estos instrumentos no convencionales. En nuestro caso, el ordenamiento jurídico monetario dominicano, ante una situación excepcional de crisis o dificultad económica de alta relevancia, que amerite su uso, tanto de forma preventiva como reactiva.

Pretendemos hacer un aporte a los diseñadores y ejecutores de la política monetaria y a la amplia doctrina del derecho de la regulación monetaria; también, ofrecemos las bases para poder hacer compatible los instrumentos no convencionales con nuestra Ley 183-02 Monetaria y Financiera, de fecha 3 de noviembre de 2002 (en adelante, "LMF"), y así permitir una visión diferente de los instrumentos convencionales que durante años hemos aplicado.

\section{Política monetaria no convencional o no ortodoxa}

Las políticas monetarias no convencionales son medidas no habituales de ejecución de la política monetaria o, lo que es lo mismo, se aplican en situaciones excepcionales, cuando es necesario generar un impacto agresivo en la economía. Se han aplicado en los casos en los que las políticas monetarias convencionales se han agotado (Blanchard et al., 2013); en 
definitiva, se trata de medidas de alto impacto y de aplicación por un largo plazo (Vérgez, 2015).

Conviene precisar que la política monetaria no convencional tuvo su génesis en la crisis financiera asiática de 1997 y fueron implementadas por el Banco Central de Japón, luego fueron aplicadas de forma más precisa en la crisis financiera de 2008 (L'éco en Bref, 2019); también, hubo algunos casos de medidas de política monetarias no estándares, como las tasas de interés negativas.

La crisis financiera de 2008 generó dificultades económicas que llevaron al agotamiento de las políticas monetarias convencionales. Este agotamiento de los instrumentos convencionales de política monetaria se debió a varios factores, entre los que podemos destacar: i) la quiebra sucesiva de importantes EIF norteamericanas y europeas que desplomaron el mercado monetario; ii) los tipos de referencias de la Fed, el BCE, el Banco de Inglaterra, el Banco Central de Suiza y el Banco de Japón, estaban próximo a cero o en cero, de manera que no se podían aumentar ni disminuir más; iii) la quiebra de tantos bancos de alta estima respecto a su solidez provocó una crisis de confianza, por lo que los ahorrantes y empresas no tenían ningún estímulo para poner su dinero a circular; y iv) el pánico ocasionó una crisis de liquidez.

Las autoridades monetarias de Reino Unido, la UE, Japón y EE. UU. ante la imposibilidad de estimular la economía lo suficientemente fuerte como para superar la crisis mediante el empleo de políticas monetarias convencionales, se vieron en la necesidad de emplear una serie de acciones al margen de los paradigmas actuales sobre las políticas monetarias; estos fueron los instrumentos no convencionales de política monetaria: forward guidance o preaviso monetario, el quantitative easing o expansión cuantitativa, el qualitative easing o expansión cualitativa y las operaciones ilimitadas de liquidez.

Debemos tomar en cuenta que el escenario perfecto para el funcionamiento de la política monetaria convencional requiere que el tipo de interés oficial no sea negativo. La razón la expresa el destacado economista John Maynard Keynes, al indicar que los agentes económicos pueden en cualquier momento preferir la liquidez absoluta cuando el rendimiento es cero (Keynes, 1936). Por lo que, cuando tenemos un tipo referencial en negativo la política monetaria convencional es ineficiente y se produce 
una trampa de liquidez, y la autoridad monetaria no puede estimular la economía con la política monetaria convencional (Princeton University Press, s. f.).

Cuando estamos frente a una trampa de liquidez es posible que las AMF realicen una política monetaria expansiva, debido a que la disminución del tipo de interés referencial no logra impulsar la demanda agregada. El término demanda agregada se entiende como la demanda total de bienes y servicios del consumidor final en un período de tiempo concreto (Sexton y Fortuna, 2005) o, dicho de otra manera, "es la relación entre la cantidad de producción demandada y el nivel de precios agregado. Nos permite determinar la cantidad de bienes y servicios que las personas desean comprar a un determinado precio" (Mankiw, 2010, p. 269). En este escenario la política monetaria pierde su impacto por tener una tasa en cero (Krugman, 1998) y no puede influir en la inversión y el consumo que son componentes de la demanda agregada.

Resulta interesante enfatizar que a pesar de que las políticas monetarias no convencionales se originaron como consecuencia de la crisis del 2008, esto no debe interpretarse en el sentido de que su aplicación solo puede efectuarse en el marco de una crisis. No obstante, se estila que para su aplicación debemos estar ante un agotamiento de la política monetaria convencional, y para que esto ocurra debemos estar en presencia de un problema económico de cierta relevancia.

En relación con lo anterior, entendemos que la dificultad crediticia o financiera que se pretenda corregir con política monetaria no convencional debe suponer al menos un riesgo de crisis o un aumento de la inflación no manejable con los instrumentos ortodoxos; o, simplemente, aplicarse cuando las circunstancias las justifican (La Finance Pour Tous, 2015). Además, por el alto volumen de masa monetaria que implica la ejecución de la política monetaria, nos parece apropiado limitar jurídicamente su uso, sin desconocer la discrecionalidad técnica que requiere la autoridad monetaria.

Las políticas monetarias no convencionales varían conforme a las particularidades de las economías de los países donde fueron aplicadas; así, la implementación y finalidad de estas importantes herramientas no convencionales de transmisión de la política monetaria se describen de forma práctica y general en la siguiente tabla: 


\section{Tabla 1. Implementación de las políticas monetarias no convencionales en EE. UU., Japón y Europa poscrisis financiera de 2008}

\begin{tabular}{|c|c|c|c|}
\hline $\begin{array}{c}\text { Bancos } \\
\text { Centrales }\end{array}$ & Medidas & Forma de Ejecución & Finalidad \\
\hline \multirow{5}{*}{$\begin{array}{l}\text { Reserva } \\
\text { Federal }\end{array}$} & \multirow{4}{*}{$\begin{array}{l}\text { Quantitative } \\
\text { Eeasing }\end{array}$} & QE1 & $\begin{array}{l}\text { Compra masiva de instrumentos: deuda } \\
\text { pública, préstamos directos y acciones, con el } \\
\text { objetivo de mejorar el crédito. }\end{array}$ \\
\hline & & QE2 & $\begin{array}{c}\text { Comprar bonos con vencimiento máximo de } \\
\text { tres ańos, con el objetivo de comprar deudas } \\
\text { sin incrementar el balance que mantiene la } \\
\text { cartera de la Fed. }\end{array}$ \\
\hline & & QE3 & $\begin{array}{l}\text { Comprar activos bancarios respaldados por } \\
\text { hipotecas de alto riesgo y comprar bonos del } \\
\text { Tesoro: nuevo intento de aumentar la liquidez. }\end{array}$ \\
\hline & & $\begin{array}{l}\text { Programa (MMIFF) y } \\
\text { las compras directas en } \\
\text { el mercado de pagarés } \\
\text { de empresas (CPFFF) }\end{array}$ & $\begin{array}{l}\text { Dar a los fondos mutuos del mercado mone- } \\
\text { tario y a otros inversionistas del mercado, } \\
\text { la confianza para realizar inversiones a largo } \\
\text { plazo, lo que contribuiría a mejorar el nivel de } \\
\text { liquidez de las EIF. }\end{array}$ \\
\hline & $\begin{array}{l}\text { Facilidades } \\
\text { de Crédito }\end{array}$ & $\begin{array}{c}\text { Programas TALF, CPFF } \\
\text { y AMLF }\end{array}$ & $\begin{array}{l}\text { Adquirir valores de renta fija de agencias feder- } \\
\text { ales y bonos de titularización hipotecaria. }\end{array}$ \\
\hline $\begin{array}{l}\text { Banco } \\
\text { Central de } \\
\text { Japón }\end{array}$ & $\begin{array}{l}\text { Operaciones } \\
\text { de mercado } \\
\text { abierto }\end{array}$ & Compra de títulos & $\begin{array}{l}\text { Crear anticipaciones de inflación que harían } \\
\text { bajar las tasas reales. } \\
\text { Asegurar un refinanciamiento abundante y } \\
\text { buen mercado bancario } \\
\text { Ayudar al financiamiento de la economía } \\
\text { Limitar la apreciación del yen. }\end{array}$ \\
\hline \multirow{6}{*}{$\begin{array}{l}\text { Banco } \\
\text { Central } \\
\text { Europeo }\end{array}$} & \multirow{4}{*}{$\begin{array}{l}\text { Medidas de } \\
\text { Apoyo al } \\
\text { crédito }\end{array}$} & Facilidades Permanentes & $\begin{array}{l}\text { Realizar subastas semanales a tipo de interés } \\
\text { fijo. }\end{array}$ \\
\hline & & Operaciones Swap & $\begin{array}{l}\text { Se pactó el suministro de liquidez en dólares } \\
\text { con la Fed a corto plazo para suplir las necesi- } \\
\text { dades de dólares. }\end{array}$ \\
\hline & & LTRO y TLTRO & $\begin{array}{l}\text { Préstamos a la banca con un interés de } 1 \% \text {. } \\
\text { Reactivar la oferta de crédito entre los bancos, } \\
\text { es decir, en el mercado interbancario. }\end{array}$ \\
\hline & & $\begin{array}{l}\text { Activos de garantía y } \\
\text { entidades de contra- } \\
\text { partida }\end{array}$ & $\begin{array}{c}\text { Admitir una mayor cantidad de activo para } \\
\text { realizar operaciones de crédito. Se integra el } \\
\text { Banco Europeo de Inversión como entidad de } \\
\text { contrapartida. }\end{array}$ \\
\hline & \multirow{2}{*}{$\begin{array}{c}\text { Otros } \\
\text { programas }\end{array}$} & $\begin{array}{l}\text { Programa para el } \\
\text { mercado de valores }\end{array}$ & $\begin{array}{c}\text { Autorizar a los bancos de la zona euro a } \\
\text { comprar instrumentos de renta fija, negociables } \\
\text { en los mercados primarios y secundarios, para } \\
\text { aumentar la liquidez del mercado. }\end{array}$ \\
\hline & & $\begin{array}{l}\text { Programa de Adquisi- } \\
\text { ciones directas en el } \\
\text { mercado de garantía }\end{array}$ & $\begin{array}{c}\text { Comprar activos de renta fija a largo plazo para } \\
\text { aumentar la liquidez de los sectores públicos y } \\
\text { privados. }\end{array}$ \\
\hline
\end{tabular}




\begin{tabular}{|c|c|c|c|}
\hline $\begin{array}{c}\text { Bancos } \\
\text { Centrales }\end{array}$ & Medidas & Forma de Ejecución & Finalidad \\
\hline \multirow{1}{*}{$\begin{array}{c}\text { Banco de } \\
\text { Inglaterra }\end{array}$} & $\begin{array}{c}\text { Líneas Swap } \\
\text { Fachet }\end{array}$ & $\begin{array}{c}\text { Líneas Swap con la Fed } \\
\text { Repos en dólares }\end{array}$ & Cubrir la demanda de liquidez en dólares. \\
\cline { 2 - 5 } & \begin{tabular}{c} 
Corporate Bond, Gilt \\
Purchases, Commercial \\
Paper Facility (CPF) \\
\cline { 2 - 5 }
\end{tabular} & $\begin{array}{c}\text { Pecured Commercial } \\
\text { Paper (SCP) }\end{array}$ & $\begin{array}{c}\text { Lograr la adquisición de activos financieros } \\
\text { privados con el marcado interés en generar } \\
\text { liquidez. }\end{array}$ \\
\cline { 2 - 5 } & $\begin{array}{c}\text { Otras } \\
\text { medidas }\end{array}$ & $\begin{array}{c}\text { Special Liquidity Scheme, } \\
\text { OMA, Facilidades } \\
\text { permanentes, Discount } \\
\text { Window Facility (DWF) }\end{array}$ & $\begin{array}{c}\text { Incrementar la liquidez del sector financiero, } \\
\text { aumentar el volumen de los OMA, facil- } \\
\text { itar a las empresas de construcción y al sector } \\
\text { bancario la adquisición de activos de calidad. }\end{array}$ \\
\hline
\end{tabular}

Fuente: elaboración propia.

\section{La política monetaria no convencional en la actual crisis económica}

La política monetaria no es estática: se va adecuando a los retos económicos; así, el Gran Confinamiento ha impulsado a la Fed, el BCE, el Banco de Inglaterra, el Banco Central de Japón, el Banco Central de Australia, entre otros, a tomar una serie de medidas de política monetaria que le permitan mantener la estabilidad económica en un escenario sin precedente. Dentro de esas medidas está la política monetaria no convencional.

Las autoridades monetarias - que implementaron políticas monetarias no convencionales en la crisis de 1998 y en la crisis financiera de 2008 - cuentan con una reconocida experiencia para llevar su aplicación a un nivel más alto; es precisamente lo que está ocurriendo en el escenario actual: los bancos centrales están llevando la aplicación de esos instrumentos a una escala sin precedente, incluso a ámbitos inexplorados.

La Fed ha sido la más innovadora en la aplicación de los instrumentos no convencionales - especialmente la flexibilización cuantitativa- en relación con su aplicación en la crisis de 2008: sobre todo en el aspecto de plazos, tipo de activos y destinatarios - mercado de bonos corporativos(Condon y Torres, 2020). Estas innovaciones no solo se deben al tamaño de la economía estadounidense, sino al objetivo monetario de la Fed, que le da un amplio margen de maniobra, con relación a otros bancos centrales. 
Así las cosas, en marzo del año 2020 la Fed anunció una serie de medidas, dentro de la cuales están: i) Reducción de su tasa de interés de referencia a cero y cero puntos veinticinco; ii) un programa de $\mathrm{QE}$ para comprar activos por valor de $\$ 700$ mil millones de dólares (bonos del Tesoro y valores respaldados por hipotecas); iii) reducción de la tasa de emergencia en la ventanilla de descuento para las EIF de 125 a 0.25 y el plazo de los prestamos fue extendido a 90 días; iv) acuerdos con el Banco de Inglaterra, el Banco Central Europeo, el Banco de Canadá, el Banco Nacional de Suiza, el Banco Central de Japón, entre otros, para mejorar y mantener la liquidez del dólar y bajar las tasas de préstamos de líneas de intercambios.

Posteriormente, la Fed anunció medidas adicionales para seguir impulsando la economía (Fed, 2020): i) anunció que mantendría y extendería su programa de compra de activos en las cantidades necesarias para respaldar el buen funcionamiento del mercado y la transmisión efectiva de la política monetaria; ii) compra de bonos corporativos comprando valores de grado de inversión en mercados primarios y secundarios por medio de fondos negociados en bolsa; iii) programa de préstamos por US300 mil millones para apoyar el flujo de crédito a los empleadores, consumidores y empresas por medio del programa Main Street; iv) ampliación del servicio del mercado monetario para incluir una gama más amplia de valores que acepta; v) expansión del fondo de financiación del papel comercial (incluyendo papel comercial exento de impuestos de alta calidad; y, vi) diminución de la tasa de repos de 0 a 0.1 .

La más relevante de todas estas medidas es su amplio programa de QE que según lo indicó la Fed —estaba preparada para usar su gama completa de herramientas para apoyar el flujo de crédito a los hogares y las empresas-. Esto quiere decir que, la Fed planea extender el QE como nunca en la historia. Adicionalmente, Jerome Powell, presidente de la Fed, indicó que "la Fed mantendría su tasa de referencia en negativo hasta que la economía haya resistido los últimos acontecimientos y esté en camino de alcanzar nuestras metas máximas de empleo y estabilidad de precios" (Laborde, 2020, párr. 6). La Fed según Chris Rupkey, economista financiero jefe de MUFG Union Bank, "está pasando de ser no solo el prestamista de último recurso, sino que ahora es el comprador de último recurso" (Cox, 2020, párr. 15) 
En el caso de Reino Unido, el Banco de Inglaterra, por medio de su Comité de Política Monetaria (MPC por sus siglas en inglés), implementó una serie de medidas económicas para mitigar los efectos de la crisis (Bank of England, 2020): i) diminución del tipo de referencia a $0.25 \%$; ii) un nuevo esquema de financiamiento a plazo con incentivos adicionales para las pequeñas y mediana empresa (TFSME, por sus siglas en inglés), financiado por la emisión de reservas del banco central; iii) el mantenimiento del stock de libras esterlinas para compras de bonos corporativos con grado de inversión no financiero, financiadas por la emisión de reservas del banco central, en $£ 10$ mil millones. iv) el mantenimiento del stock de compras de bonos del gobierno de Reino Unido, financiado por la emisión de reserva del banco central en $£ 435$ millones. v) reducción de la tasa de amortiguación de capital anticíclica del Reino Unido al 0 \% de las exposiciones de los bancos a los prestatarios de Reino Unido.

De todas estas medidas es preciso resaltar que el Banco de Inglaterra ha implementado medidas de flexibilización cuantitativa, al comprar bonos del gobierno con el respaldo de la reserva del banco central. Estas compras se hacen conjuntamente con una reducción del tipo de interés en $0,25 \%$ (Elliott, Davies, Partington, 2020); también, se aprecia un financiamiento del Banco de Inglaterra al gobierno por medio de la compra de deuda pública, esto es gracias a que la referida entidad monetaria no tiene las mismas restricciones de otros bancos centrales como el BCE, pues está directamente involucrado en las finanzas del gobierno (Milliken, 2020). Sin embargo, el Tesoro y el Banco de Inglaterra indicaron que cualquier uso de la instalación por parte del gobierno para ayudar a las empresas y los trabajadores sería temporal y a corto plazo (BBC News, Ping, 2020).

El Banco Central Europeo también adoptó una serie de medidas de política monetaria que, en sentido general, se encaminaron a mantener la liquidez del sistema financiero y la estabilidad macroeconómica de la zona euro; dentro de esas medidas se pueden destacar las siguientes: i) se realizaron operaciones de refinanciamiento de largo plazo (Long Term Refinancing Operations, en lo adelante "LTRO") con la finalidad de proporcionar liquidez al sistema financiero; ii) compra de activos netos adicionales de $€ 120$ mil millones hasta el final del año, con la finalidad de estimular la compra de activos del sector privado; iii) las tasas de 
interés de las principales operaciones de refinanciamiento serán de $0.00 \%$, las tasas de interés de la facilidad marginal de crédito serán de $0.25 \%$ y la facilidad de depósito se mantendrá en $-0.50 \%$.

El BCE también tomó medidas agresivas de flexibilización cuantitativa: i) un nuevo programa de emergencia pandémica (PEPP) de compra de activos temporales de valores del sector público y privado por un monto de $€ 750$ mil millones —incluyendo una categoría de activos admisibles más amplia—; ampliación del rango de activos elegibles bajo el programa de compras del sector corporativo (CSPP) a papel comercial no financiero; facilitar los estándares de garantía mediante el ajuste de los principales parámetros del marco de garantía.

De la misma forma que la Fed el BCE anunció por conducto del Consejo de Gobierno que estaba dispuesto a seguir aumentando el tamaño de sus programas de compra de activos y ajustar su composición, tanto como sea necesario y durante el tiempo que sea necesario (BCE, 2020).

El Banco de Reserva Australiano en lo adelante "RBA" también adoptó medidas de política monetaria para hacer frente a la crisis económica que provocó la pandemia del Covid-19. Entre sus principales medidas se encuentran: i) disminución del tipo de referencia $0,25 \%$; ii) apuntar al rendimiento de los bonos del gobierno del 0,25\% para ayudar a reducir los costos de financiación en toda la economía; iii) compra de bonos del gobiernos australiano en el mercado secundario; iv) proporcionar un servicio de financiación a plazo para el sistema bancario para apoyar los préstamos de empresas; v) proporcionar liquidez al sistema financiero mediante la realización de operaciones diarias al mercado y con operaciones regulares de recompra de vencimiento de uno, tres y seis días; vi) proporcionar liquidez al mercado de bonos del gobiernos mediante la compra de bonos del gobierno australiano y valores semi-gubernamentales (RBA,2020).

El Banco Central de Japón también ha implementado políticas monetarias no convencionales: especialmente el QE. Así, ha dispuesto la compra ilimitada de deuda pública y de bonos empresariales (expandió el límite de compra de deuda corporativa hasta 20 billones de yenes), como forma de mantener la estabilidad macroeconómica; también, expandió su programa de préstamos de emergencia a una mayor cantidad de instituciones de intermediación financiera (El País, 2020). 
Del resumen de las medidas tomadas por los principales bancos centrales descritas, se infiere que, aunque no estamos ante una crisis financiera, la política monetaria no convencional sigue jugando un papel estelar en el mantenimiento de la estabilidad macroeconómica en tiempo de crisis. Ahora, su aplicación ha sido a niveles sin precedentes, pues ya han mostrado su efectividad cuando los instrumentos de política monetaria convencional no pueden generar el impulso necesario a la economía.

En el plano local, el Banco Central de la República Dominicana también tomó una serie de medidas de política monetaria para mantener la estabilidad macroeconómica del país, dentro de las cuales se pueden mencionar las siguientes: facilidades de liquidez a las EIF por RD\$ $50 \mathrm{mil}$ millones, mediante repos a 90 días y tasas de $5 \%$ anual (garantizados con valores de Hacienda y del Banco Central); liberalización de $\mathrm{RD}$ \$30,133.4 millones del encaje legal para la canalización de nuevos préstamos (se flexibilizó la garantía sustituyéndose valores de Hacienda y del Banco Central por los contratos de los préstamos otorgados); facilidades de liquidez en moneda extranjera para las EIF por US\$ 400.0 millones mediante repos a 90 días con una tasa de $1.80 \%$ anual, adicionalmente, se liberó del encaje legal US\$ 222.4 millones para canalizar divisas a los sectores productivos con garantía en los contratos de préstamos otorgados.

Otras medidas adicionales fueron: la reducción del coeficiente de encaje legal a 0.5 para los bancos de ahorro y crédito y para las corporaciones de crédito, lo que representa una liberación del encaje legal de RD\$ 136.4 millones; liquidez en dólares por US\$ 662.4 al mercado cambiario, de los cuales US\$ 400 se otorgarían mediante repos a 90 días y US\$ 222.4 de liberación del encaje legal en moneda extranjera para impulsar nuevos préstamos a los sectores productivos; disminución de la tasa de interés de política monetaria (TPM) de 4.50 a 3.50 anual y la tasa de interés de depósitos overnight de $3.0 \%$ a $2.50 \%$ anual, la de repos a 1 día de $6.0 \%$ a $4.50 \%$ anual y la de repos hasta 90 días se estableció en $5.0 \%$ anual.

Las medidas adoptadas por el Banco Central no constituyen medidas de política monetaria no convencional, sin embargo, en algunos casos se hicieron algunas flexibilizaciones, especialmente en el tipo de garantía. De esto se infiere que los instrumentos convencionales fueron suficientes para transmitir la política monetaria, sin embargo, es de vital importancia 
explorar otros mecanismos de transmisión de la política monetaria en contextos de crisis económica.

\section{Potestad de la AMF para diseńar y ejecutar nuevos instrumentos de política monetaria}

La Constitución no indica de forma expresa los límites en el diseño e implementación de la política monetaria; por el contrario, lo deja como una reserva legislativa: no obstante, es preciso indicar que la misma destaca que la regulación del sistema monetario y financiero es facultad de la Junta Monetaria, como órgano superior del BCRD (artículo 223).

En este propósito, el primer acercamiento a los límites legales se infiere de lo dispuesto por el artículo 3 literal (d) de la LMF que, a grandes rasgos, expresa que la AMF deberá garantizar el funcionamiento del sistema monetario con la aplicación de instrumentos de política monetaria, conforme a la referida LMF y las normas y prácticas internacionales.

Dicho lo anterior, es necesario ir de inmediato al punto central de la cuestión; así tenemos que, la Junta Monetaria tiene la facultad discrecional de implementar cualquier instrumento de ejecución de política monetaria, siempre que sea en operación de mercado y de forma indirecta, conforme se indica en el artículo 26 literal (c) de nuestra LMF.

En tal sentido, se concluye que todo instrumento de ejecución de política monetaria que se pretenda implementar debe, en primer lugar, cumplir con la condición de que sea de forma indirecta y de mercado abierto. Cuando habla de forma indirecta, quiere decir que el BCRD cuando lo ejecute no debe hacer operaciones directamente con el público, sino por medio del mercado secundario.

Pese a lo que indica el artículo 26 literal (c) de la Junta Monetaria, conviene mencionar a modo de referencia la Resolución de la Junta Monetaria No. 030403-CO, de fecha 4 de marzo de 2003, mediante la cual emite un comunicado sobre los certificados de inversión emitidos al público por 5 millones de pesos (Junta Monetaria [JM], Resolución No. 030403, 2003). En este documento la Junta Monetaria indica que el BCRD establece que la venta directa al público no infringe las disposiciones de la LMF y fundamenta su postura en el artículo 26 literal (c), el cual opone al artículo 26 literal (a). 
De forma más precisa, la Junta Monetaria lo que planteó fue que esta tenía la posibilidad, con el voto de la $2 / 3$ parte de sus miembros, de establecer otro instrumento de ejecución de la política monetaria. Por lo tanto, no interfiere con lo aducido por economistas, juristas y estudiantes al indicar que "el BCRD podrá realizar operaciones de mercado abierto exclusivamente con EIF y con inversionistas institucionales" (JM, Resolución No. 030403, 2003, p. 1).

En medio del debate la Junta Monetaria indicó lo siguiente: i) Los bancos centrales tienen el derecho de establecer el precio al que venden sus productos, como lo hace la Fed o el Tesoro de EE. UU.; ii) que los personas están en la libertad plena de decidir si adquieren o no estos productos, de manera que el $\mathrm{BCRD}$ no le impone directamente la obligación de adquirirlos; iii) que el FMI sugirió al BCRD la colocación de certificado de participación al público.

Lo antes indicado generó muchas opiniones contrarias de economistas y juristas destacados, aduciendo que la LMF es bastante clara al establecer los límites jurídicos que deben ser tomados en cuenta al momento de establecer nuevos mecanismos de ejecución de la política monetaria. En ese sentido, se argumentó que el legislador dejó la potestad discrecional a la Junta Monetaria para decidir implementar con el voto de $2 / 3$ partes de sus miembros cualquier instrumento de política, fue muy sabio y previsor al no dejar esta posibilidad completamente abierta.

La postura de la Junta Monetaria expresada en la citada resolución de 2003, es el criterio oficial para los fines del tema que venimos exponiendo en esta sección. De manera que, podemos inferir que existe una flexibilización parcial de los límites jurídicos que tiene la Junta Monetaria para implementar otros instrumentos de ejecución de la política monetaria no previstos en la ley.

Es necesario indicar que las operaciones directamente con el público, según se interpreta en la indicada resolución de 2003, no pueden hacerse de forma ordinaria. Es decir, están supeditadas a la existencia de una situación excepcional que amerite su implementación. Ese mismo criterio se evidencia en la parte principal del artículo 27 literal (a) del proyecto de modificación de la LMF, establecido textualmente "en situaciones excepcionales que lo ameriten". 
La operación directa con el público fue impensable antes del 2003, por lo que hay que tomar como referencia el contexto en el que se aplicaron. Para el año 2003, en nuestro país ocurrió la quiebra del Banco Intercontinental (BANINTER), el banco comercial más importante del país en esos momentos; así como también, Mercantil y el Nacional de Crédito (BANCRÉDITO).

La quiebra de estos bancos contribuyó al surgimiento de la crisis financiera de 2003 (Jiménez, 2019) que impactó en aspectos económicos, políticos y sociales nuestro país. Precisamos de una vez que, en la opinión de afamados economistas nacionales, la crisis causada por la quiebra de los bancos se debió a una deficiencia regulatoria y una escasa supervisión prudencial por parte de las autoridades, matizada por la existencia de tráfico de influencias que de cierta forma impedía una regulación imparcial, oportuna y eficaz (Montas, s.f.).

En este punto, debemos esclarecer que nuestro objetivo no es estudiar la crisis financiera de 2003, sino relacionar esta con la decisión de la Junta Monetaria de realizar operaciones directas con el público. De tal modo que resulta necesario preguntarnos: ¿̇se trató de una medida no convencional de política monetaria?

Para responder la cuestión debemos partir de la naturaleza de las políticas monetarias no convencionales. Si decimos que los instrumentos no convencionales de política monetaria son excepcionales, a largo plazo, que buscan generar un gran impacto en la economía, y responden a una dificultad económica que los instrumentos monetarios convencionales no pueden resolver, pudiéramos decir que sí.

A pesar de lo último señalado, la Junta Monetaria es el organismo superior de la AMF, cuyas decisiones expresadas a través de actos administrativos gozan de una presunción de validez, como se establece en el artículo 4 literal (a) de la LMF. En ese mismo sentido, conviene agregar que no serán susceptibles de recursos las decisiones de la Junta Monetaria que defina el objetivo anual de la programación monetaria, las de ejecución de la política monetaria y las que aprueben o modifiquen el plan anual de inspección y supervisión financiera, como se precisa en el artículo 4 literal (d) de nuestra LMF.

Conviene aclarar que la irrecurribilidad de las decisiones de la Junta Monetaria, más arriba citada, no es absoluta; pues si bien la AMF 
goza de un margen amplio de discrecionalidad técnica, esta no puede emitir decisiones que sean manifiestamente contraria al derecho. En ese sentido, podemos agregar que existe un control jurídico sobre el respeto al debido proceso al dictar el acto y un control jurídico sobre la razonabilidad (Prats y Victoria, 2008).

En el marco de lo antes indicado, Prats y Victoria, 2008 precisan que "el control de la razonabilidad implica un control de constitucionalidad, debido a que todo lo razonable es constitucional" (p. 110) y, por tanto, jurídicamente admisible en un Estado de Derecho (Tomás, 1994). Siguen diciendo que el juez debe someter las actuaciones administrativas, en este caso de la Junta Monetaria, a un control de bloque de constitucionalidad que se compone de "principios, normas y una serie de valores como el orden, la paz, la seguridad, la igualdad, la justicia, la libertad y otros que, al ser asumidos por nuestro ordenamiento jurídico, se configuran como patrones de razonabilidad" (Suprema Corte de Justicia, 4 de agosto de 2004)

Refiriéndonos a la delimitación de las fronteras de las actuaciones de la AMF en sentido amplio, es preciso indicar que la AMF dominicana tiene una política monetaria basada en la estabilidad de precios. Esta afirmación se fundamenta en la parte in fine del artículo 228 de la Constitución Dominicana y en el artículo 2 literal (a) de la LMF, cuando indica que "la regulación del sistema monetario tendrá por objeto mantener la estabilidad de precios, la cual es base indispensable para el desarrollo económico nacional".

En ese mismo sentido, el artículo 15 de la referida ley indica que:

El Banco Central tiene por función de ejecutar las políticas monetaria, cambiaria y financiera, de acuerdo con el Programa Monetario aprobado por la Junta Monetaria y exclusivamente mediante el uso de los instrumentos establecidos en el Título II de esta Ley, conforme a los objetivos establecidos en el Artículo 2, literal (a).

Es necesario aclarar que lo establecido por el artículo 15, anteriormente citado, no debe interpretarse en el sentido literal, pues ya hemos establecido al principio que la Junta Monetaria puede implementar otros instrumentos monetarios. Por consiguiente, entendemos que la 
exclusividad de los instrumentos monetarios establecidos en el Título II de la LMF no se refiere a una limitación estricta.

Haciendo una interpretación jurídicamente adecuada de la relación del artículo 15 con el artículo 26 literal (c) de esta LMF, se puede concluir que lo que busca el legislador es que no se ejecute la política monetaria por medio de instrumentos no previstos en la ley ni tampoco establecidos por la Junta Monetaria. Lo anterior tiene su fundamento en lo que indica el artículo 3 literal (d) en su parte in fine, en el sentido de que la AMF ejecutará la política monetaria con apego a la LMF y las normas y prácticas internacionales sobre la materia.

La otra parte que debemos enfatizar es lo que establece la parte in fine del artículo 15 cuando indica que el uso de los instrumentos monetarios debe responder al objetivo de la AMF descrito en el artículo 2 literal (a), que es "mantener la estabilidad de precios, la cual es base indispensable para el desarrollo económico nacional". Lo anterior puede considerarse, a nuestro entender, como un límite a la aplicación de otros instrumentos de política monetaria. Supeditar a que los instrumentos respondan al fin de la AMF es conveniente porque delimita las fronteras de la potestad discrecional técnica de las autoridades monetarias.

El artículo 33 de la LMF establece otros límites importantes, como el hecho de que el BCRD solo pueda prestar dinero a las EIF en su calidad de prestamista de última instancia, es decir, solo podrá prestar recursos cuando estas entidades no lo encuentren por otra vía (mercado interbancario) y siempre que sea en situaciones de iliquidez temporal. Además, el mismo artículo prohíbe que el BCRD pueda financiar de forma directa o indirecta al gobierno, salvo casos excepcionales.

Por otra parte, el Banco Central tiene que ejecutar la política monetaria conforme al programa monetario. Esto nos resulta interesante porque el programa monetario se publica al inicio de cada ańo y se revisa trimestralmente. El simple hecho de hacer pública la política monetaria ya crea una situación de previsión del comportamiento de esta por los sectores de la economía (mercado financiero, mercado de valores, mercado de seguros, etc.). Es posible afirmar lo anterior en vista del artículo 26 de la LMF, que dispone que el programa monetario debe contener "en forma explícita los 
objetivos y metas que se persigan para el período de que trate, así como las medidas o acciones de política que se estimen necesarias para asegurar su cumplimiento".

Continuando con lo antes expresado, destacamos que los instrumentos de política monetaria deben también responder a los objetivos del programa monetario, y eso puede constituir una condición para su uso. Esto también garantiza que la política monetaria sea creíble y que por tanto genere confianza en los sectores económicos. La importancia de este aspecto se revela en que el legislador atribuyó a la Junta Monetaria, no solo la facultad de aprobar el programa monetario, sino también de conocer y fiscalizar su grado de ejecución, como se observa en el artículo 9 literal (b) de la LMF.

\section{Implementación de las políticas monetarias no convencionales conforme a la LMF}

Nuestra LMF no ha establecido los instrumentos de política monetaria descritos en el artículo 26 como numerus clausus. Por el contrario, se deduce de la lectura combinada de los artículos 3 literal (d) y 26 literal (c) que es numerus apertus, aunque esto último este jurídicamente limitado, incluso a situaciones excepcionales. Tomando en cuenta esta aclaración, vamos a someter cada instrumento de política monetaria no convencional a las particularidades de nuestra legislación para fundamentar la posibilidad que tenemos de utilizarlos.

El instrumento no convencional que menos problemas de compatibilidad jurídica presenta es el "forward guidance" que es español se traduce como "preanuncio monetario"(FUNDEU BBVA, 2015). Este instrumento, al cual nos hemos referido previamente, no tiene ninguna restricción legal que impida su aplicación en nuestro ordenamiento jurídico monetario, debido a que informar previamente las actuaciones de la autoridad monetaria es muy recomendado - dependiendo de la coyuntura económica-, pues sirve para la señalización de la política monetaria, lo que determinará su efectiva transmisión.

La autoridad monetaria no puede sorprender a los agentes económicos con la ejecución repentina de instrumentos monetarios, ya que esto 
puede causar dificultad en la recepción de la misma, obteniendo resultados diferentes a los buscados. Sin embargo, no es lo mismo informar a los agentes económicos cuando se trata de una política monetaria ordinaria, que cuando los agentes económicos tienen pánico tras los efectos de una crisis. Conviene agregar que el forward guidance busca además de informar, hacer creíble y confiable la política monetaria, al hacer una explicación precisa de la trayectoria futura del tipo de referencia (Den, 2013, pp. 1-21) a medio plazo.

En ese orden y dirección, nuestra normativa prevé el Programa Monetaria, que es definido por el Reglamento sobre el Programa Monetario e Instrumentos de Política Monetaria como modelo macroeconómico (artículo 4, literal gg), que tiene por objetivo establecer la estrategia anual que seguirá el BCRD para mantener la estabilidad de precios (artículo 7, literal b).

En nuestra opinión, tiene también una finalidad informativa, pues el mismo debe ser publicado para garantizar la transparencia (artículo 12 del indicado reglamento). Pese a que no es un instrumento de ejecución de política monetaria, se asemeja mucho al objetivo del forward guidance, lo que nos indica que este pudiera ser perfectamente implementable en nuestro ordenamiento jurídico.

En lo que respecta al "quantitative easing (QE)" o "expansión cuantitativa", en español, previamente explicado, destacamos que este se puede utilizar de forma matizada, pues su naturaleza no es enteramente incompatible con nuestra LMF. Nótese, que la expansión cuantitativa tiene por característica la estimulación de la economía mediante la compra de deuda pública y del sector privado. El objetivo de usar este instrumento es inyectar capital de forma masiva para estimular a las EIF a aumentar el crédito. Esta compra de activos a gran escala supone un aumento del balance del banco central, pero no implica un cambio de la composición de los activos.

La compra de bonos de deuda pública es un instrumento actualmente practicable, pues nuestra LMF lo ha permitido en el marco de las operaciones de mercado abierto, como se puede evidenciar en el artículo 26 literal (a). No obstante, existen condiciones para su utilización que se observan en la parte in fine del artículo antes citado. Se requiere que: i) la compra se haga exclusivamente en el mercado secundario, ii) que se trate 
de títulos emitidos por lo menos (1) año antes de la compra. Estas condiciones no aplican cuando se trata de los casos previstos en el artículo 16 literal (e) y artículo 82 relativo a la distribución del superávit o déficit y deudas y déficit de operaciones, respectivamente.

Llama nuestra atención que se requiera la ejecución de este instrumento en un mercado secundario, porque esto evidencia el interés de que los instrumentos de política monetaria sean indirectos y de mercado. En este sentido, vemos una dificultad jurídica, y es que nuestra normativa exige que por lo menos exista un (1) año entre la emisión del bono de deuda pública y su compra. Esto podría generar dificultades si en una situación de crisis se requiere comprar bonos de deuda pública de forma masiva y estos no hayan cumplido con el plazo mínimo de un año.

Por otro lado, el artículo 33 de la LMF prohíbe la financiación del gobierno tanto de forma directa como indirecta. Sin embargo, esta limitante no es definitiva, debido que la autoridad monetaria con el voto de las 2/3 parte de sus miembros puede conceder crédito exclusivamente al gobierno central por medio de préstamos o adquisiciones de bonos, valores o documentos representativos de deuda, sujeto al cumplimiento de los siguientes requisitos: i) que el Congreso Nacional declare por Ley el país en situación de emergencia; ii) que el financiamiento sea por medio de entidades de intermediación financiera; iii) que la tasa de interés no sea inferior a la del mercado; iv) que el monto otorgado no exceda del dos por cierto (2\%) del promedio de los ingresos del Estado.

Respecto a esta prohibición hay que hacer una ponderación entre la prohibición del financiamiento del gobierno en situaciones ordinarias, y la necesidad de implementar políticas de QE ante una situación de crisis económica, donde las herramientas convencionales se agotan. En tales circunstancias, la AMF debe apegarse a su mandato constitucional de mantener la estabilidad de precios (artículo 228 de la Constitución), como la base del desarrollo económico nacional, según se evidencia en el artículo 2 literal (a) de la LMF.

Otro punto importante es que la compra de instrumentos de deuda pública en el marco de la QE no tiene como objetivo financiar al gobierno, sino sacar del mercado esos instrumentos que en momentos de crisis son muy seguros, evitando así, que las EIF, las agencias de seguros 
y otros inversionistas puedan adquirirlo y se vean obligados a otorgar créditos, pues el objetivo del QE es generar liquidez.

La conclusión respecto al uso de la expansión cuantitativa es que este instrumento se puede usar teniendo como límites las condiciones previamente señaladas. Sin embargo, entendemos que el plazo de un ańo de antigüedad para poder comprar bonos de deuda pública pudiera ocasionar que este instrumento no tenga la magnitud requerida ante una situación excepcional que amerite un alto estímulo al mercado financiero por parte del BCRD. Esto es en razón de que la no convencionalidad de los instrumentos monetarios se debe a su magnitud y a su extenso plazo de aplicación.

El otro instrumento no convencional, que también va encaminado a aumentar la liquidez del sector crediticio, es el "qualitative easing" $o$ "expansión cualitativa" en español, que contrario a la expansión cualitativa va enfocado a incrementar o a modificar la composición de los activos del banco central sin aumentar su balance. En otras palabras, se trata de la sustitución de activos de alto valor, por activos de alto riesgo o menos líquidos.

En nuestra normativa no existe ninguna limitación que imposibilite a la autoridad monetaria a utilizar la expansión cualitativa, debido a que para adquirir activos se debe hacer mediante el mercado secundario y, al respecto, el artículo 26 literal (a) establece claramente que "tales operaciones, en cualesquiera de las modalidades habituales de mercado, se realizarán, garantizarán o se colateralizarán solamente con títulos de deuda pública o con títulos emitidos por el Banco Central, cualesquiera que sean sus términos, moneda y condiciones de emisión”. De lo citado se concluye, que la autoridad monetaria podrá implementar este instrumento sin mayores complicaciones.

Otro instrumento no convencional importante que podemos aplicar son las operaciones ilimitadas de liquidez. Estas tienen como razón de ser aumentar la cantidad de circulante en el mercado financiero para que estos aumenten los préstamos. Este instrumento fue muy aplicado por el BCE mediante las denominadas subastas rápidas, inyectando un alto volumen de dinero a los bancos en forma de préstamos por plazos entre uno y tres días. 
Sobre este aspecto, el Reglamento sobre el Programa Monetario e Instrumentos de Política Monetaria prevé la subasta de letras y repos a un (1) día. Las subastas de letras a un (1) día serán realizadas por el BCRD cuando según su proyección, el sistema bancario de forma consolidada presente excedente de liquidez durante el día (artículo 33). Mientras que, por su parte, el BCRD aplicará subastas de repos a un (1) día, cuando el sistema bancario de forma consolidada, presente faltante de liquidez durante el día, conforme a las proyecciones del BCRD (artículo 35).

Esta última figura puede ser afín a las subastas de alto volumen que se realizaron en el marco de las operaciones ilimitadas de liquidez. Sin embargo, debemos precisar que le es afín solo en una mínima parte, pues las operaciones ilimitadas de liquidez tienen otros plazos y admiten otras garantías.

Otro punto para resaltar es que el Reglamento sobre el Programa Monetario e Instrumentos de Política Monetaria prevé en sus artículos 43 y 44, la posibilidad de la colocación directa de repos de un (1) día. Este instrumento forma parte de las facilidades permanentes de contracción y expansión monetaria a un (1) día (artículo 38) y tiene por objetivo lograr que las EIF puedan obtener liquidez al final del día. De esto se concluye que nuestras autoridades monetarias prevén un instrumento que permita a las EIF obtener liquidez, semejante a las operaciones ilimitadas de liquidez que venimos planteando.

En el Reglamento sobre el Programa Monetario e Instrumentos de Política Monetaria también consta el instrumento de reporto a plazos. Mediante este instrumento el BCRD podrá realizar operaciones de reporto a plazos con entidades de intermediación financiera, cuando las EIF necesiten liquidez por un tiempo más amplio (artículo 46) y se podrán llevar a cabo por medio de subasta públicas (artículo 47). Con este instrumento el BCRD podrá proveer de liquidez al mercado financiero cuando nos encontremos ante una dificultad económica que provoque una contracción del crédito por falta de liquidez, como lo persiguen las operaciones ilimitadas de liquidez.

Nuestra normativa contiene una figura, que sin bien no es igual a las operaciones ilimitadas de liquidez, entendemos que en una situación excepcional pudiere serle afín. Se trata del prestamista de última instancia, 
que se utiliza cuando una EIF presenta una situación temporal de iliquidez no causada por problemas de insolvencia, como se precisa en el artículo 33 de nuestra LMF. Empero, debemos aclarar que el prestamista de última instancia no es un instrumento de política monetaria propiamente dicho, pese a que existen doctrinarios de la ciencia jurídica que consideran que debería serlo.

Volviendo al punto que nos ocupa, el BCRD es la última instancia a la que las EIF pueden acudir en busca de suplir una necesidad de crédito, es decir, primero deben agotar la búsqueda del crédito en el mercado interbancario. Dicho esto, recordemos que en la crisis el mercado interbancario estaba en pánico ante la debacle financiera de 2008; así, las EIF no encontraban crédito, porque ningún banco se atrevía a prestar por el miedo que existía ya que no se sabía que EIF tenía problemas por las hipotecas subprimes. Entonces, esta realidad es compatible con lo establecido en el artículo 33 literal (a) de nuestra LMF.

La dificultad se presenta en lo referente al monto de los préstamos en las operaciones ilimitadas de liquidez; esto se debe a que, en el marco de la crisis financiera, las EIF podían pedir cualquier suma siempre que tuvieran la garantía requerida y que el préstamo fuera durante el plazo establecido que podía ser de 7, 28 y 84 días, según se constata en los informes de la prensa internacional para esa época (BBC Mundo, 2007). Pero eso no ocurre en nuestro ordenamiento jurídico, pues, el artículo 33 literal (a) de la LMF restringe el monto hasta $1 \frac{1}{2} 2$ veces el capital pagado de la entidad, además, requiere una garantía que no puede ser inferior de $1 \frac{1}{2}$ veces del valor del préstamo.

Las garantías que se pueden otorgar son las siguientes: i) títulos, ii) depósitos en el Banco Central ii) cartera de bajo riesgo, iii) mediante compra de títulos con pacto de recompra o mediante compra de cartera de bajo riesgo. Finalmente, en lo que respecta al plazo es de máximo 30 días, de manera que no pueden aplicarse los programas de operaciones ilimitadas de liquidez por un plazo mayor.

Todos estos instrumentos, a pesar de que pueden ser compatibles con nuestra normativa, tienen que cumplir una condición general: se trata de la aprobación de la Junta Monetaria con el voto de $2 / 3$ partes de sus miembros, tal como lo establece el artículo 26 literal (c), además, de que su aplicación debe ser indirecta y de mercado abierto. 
El artículo anterior citado no precisa que los nuevos instrumentos que la Junta Monetaria decida aprobar y cumplan con la condición de ser indirectos y de mercado tengan que responder a una situación excepcional. En este sentido, opinamos que el criterio que debe imperar es que la Junta Monetaria entienda que su aplicación sea necesaria para cumplir con el mandato de la regulación monetaria y financiera que es "mantener la estabilidad de precios, como la base del desarrollo económico nacional”, según se evidencia en el artículo 2 literal (a).

La conclusión que se extrae de este análisis es que la posibilidad de aplicar los instrumentos no convencionales de política monetaria no debe verse como blanco o negro, por el contrario, debe entenderse como una conjugación de matices grises. En términos jurídicos, nos referimos a que estos instrumentos tienen particularidades que no son compatibles con nuestra normativa y otras que sí. Por consiguiente, la aplicación de los mismos solo puede ser posible en la medida en que sean compatibles con nuestra normativa, como hemos explicado más arriba.

\section{Implementación de las políticas monetarias no convencionales como una práctica internacional limitada a situaciones excepcionales}

Consideramos que, en lo que respecta a la aplicación de instrumentos no convencionales de política monetaria, es necesario el criterio de situación excepcional. Para esto proponemos un doble fundamento: jurídico y fáctico.

En el sentido jurídico, entendemos que no se puede dejar a la administración monetaria una facultad tan amplia que incentive el uso indiscriminado de usar políticas monetarias no convencionales para cumplir objetivos monetarios que se pueden lograr perfectamente con instrumentos convencionales. Debemos recordar lo que explicamos en el apartado de respuesta ante la crisis, es decir, las medidas no convencionales que aplicaron los países, de donde se infiere que en muchos casos se tuvo que recurrir a la alteración de instrumentos convencionales y eso de cierta forma es un alto costo para el Estado.

En el fundamento fáctico planteamos que, si no se trata de una debacle del sistema financiero como el presentado en la crisis de 2008, 
por lo menos la aplicación de los instrumentos no convencionales debe supeditarse a una situación que agote los instrumentos convencionales de política monetaria. En apoyo de nuestro argumento tenemos que los estudiosos de los instrumentos no convencionales de política monetaria opinan que se "deben aplicar de forma excepcional ante situaciones de crisis" (Gertler y Karadi, 2011, pp. 17-34; Gil, 2014, p. 349).

En este mismo orden y dirección, si partimos de la aplicación histórica de los instrumentos no convencionales, llegaremos a la conclusión de que solo se han aplicado en situaciones excepcionales, pues la primera vez que se usó un instrumento no convencional de política monetaria de gran impacto fue en Japón, como consecuencia de la trampa de liquidez en la crisis de 1997. Así lo expresan Kang y Syd (2008), antiguos economistas en el departamento de Asia y el Pacífico del FMI, al indicar que:

Por la amplitud de la crisis, y su carácter gradual, la estrategia de Japón evolucionó con el tiempo, proporcionando liquidez al principio mediante medidas innovadoras y excepcionales por parte del Banco de Japón, como la ampliación de la lista de garantías, la compra directa de activos y la aplicación de una política muy expansiva, de tasa de interés cero. (p. 24)

Según lo que explica Karube Kensuke (2011) en un diario japonés, el gobierno de Japón aprobó una ley para facilitar la inyección de dinero público con la finalidad de devolver la estabilidad económica al promover la liquidez del sistema financiero nipón. Si hacemos un símil con lo que venimos planteando y la situación presentada en la crisis de 2008, que dio origen a la aplicación de instrumentos no convencionales de política monetaria, solo podemos llegar a una conclusión. Que la naturaleza propia de estos instrumentos hace que su aplicación sea eminentemente excepcional al abordar un problema de gran magnitud (Trichet, 2010; Esteve y Prats, 2011).

\section{Conclusión}

Una vez demostrada la posibilidad de usar los instrumentos no convencionales de política monetaria, proponemos que su implementación no 
debe hacerse de forma indiscriminada. Por lo que, sugerimos, como hemos indicado más arriba, definir sus límites en función de la gravedad del problema económico a resolver.

Finalmente, debemos advertir que las políticas monetarias no convencionales están jugando un rol estelar en la crisis económica actual, pues han sido acciones muy útiles para mitigar el impacto económico que han dejado las medidas de confinamiento y distanciamiento social adoptadas en todo el mundo. Por consiguiente, entendemos que la AMF dominicana debe equiparse con las mejores herramientas de ejecución de la política monetaria, especialmente ante la incertidumbre económica que representa la pandemia del Covid-19.

\section{Referencias}

Adelina, I. (2011). Monetary Policy and Economic Policy. Titu Maiorescu University, Romania, Journal of Knowledge Management, Economics and Information Technology, ISSN 2069-5934. Recuperado de: https://www.scientificpapers.org/wp-content/files/1113_Monetary_Policy_and_Economic_Policy.pdf

Banco Central de la República Dominicana. Reglamento sobre el programa monetario e instrumentos de política monetaria, contenido en la segunda resolución de la Junta Monetaria de fecha 23 de noviembre de 2017.

Banco Central Europeo (BCE). (2020). Monetary policy decisions. Recuperado de: https://www.ecb.europa.eu/press/pr/date/2020/html/ ecb.mp200430 1 eaa128265.en.html

Bank of England. (2009). Asset Purchase Facility, APF Operating Procedures. Recuperado de: https://www.bankofengland.co.uk/-/media/ boe/files/markets/asset-purchase-facility/operatingprocedures. pdf?la=en \&hash=F076C3AB5C266D0500BE178F32C1626CA1BFACDE

Bank of England. (2020). Bank of England measures to respond to the economic shock from Covid-19. Recuperado de: https://www.bankofengland.co.uk/news/2020/march/boe-measures-to-respond-tothe-economic-shock-from-covid-19 
Blanchard, O., Dell G. \& Mauro, P. (2013). Rethinking Macro Policy II: Getting Granular. Pág. 3, International Monetary Fund, ISBN 9781484363478. Recuperado de: https://www.imf.org/external/ pubs/ft/sdn/2013/sdn1303.pdf.

Condon, C., \& Torres, C. (2020). Fed comenzará a comprar cartera de bonos corporativos de Estados Unidos. Bloomberg. Recuperado de: https://www.perfil.com/noticias/bloomberg/bc-fed-comenzara-a-comprar-cartera-de-bonos-corporativos-de-eeuu.phtml?rd=1

Constitución Dominicana, del 13 de junio de 2015. Gaceta Oficial, 10 de junio de 2015, Núm. 10805.

Cox, J. (2020). The Federal Reserve just pledged asset purchases with no limit to support markets. CNBC. Recuperado de: https://www. cnbc.com/2020/03/23/fed-announces-a-slew-of-new-programs-tohelp-markets-including-open-ended-asset-purchases.html

Den, W. (2013). Forward Guidance, Perspectives from Central Bankers, Scholars and Market Participants. Pág. 1-21. London, Centre for Economic Policy Research.

Drumetz, F., Pfister, C. \& Sahuc, J. (2015). Politique Monétaire. De Boeck Supérieur S.A., (2e ed.), Fond Jean Pâques. ISBN 978-2-80419023-1, ISSN 2030-50. Recuperado de: https://www.decitre.fr/ media/pdf/feuilletage/9/7/8/2/8/0/4/1/9782804190231.pdf

ElBancode Japón anuncia comprasilimitadas de deuda antes delas reuniones de BCE y Fed (2020). Recuperado de: https://cincodias.elpais.com/ cincodias/2020/04/27/mercados/1587973394_884974.html

Elliott, L., Davies, R. \& Partington, R. (2020). Bank of England and Treasury race to stem Covid-19 fallout" The Guardian. Recuperado de: https://www.theguardian.com/business/2020/mar/18/britain-faces-economic-emergency-warns-new-bank-of-england-governorandrew-bailey-covid-19

Esteve, V. \& Prats, M. (2011). La utilización de medidas de politica monetaria no convencional frente a la crisis financiera internacional. España. Recuperado de: https://www.uv.es/ - estevev/PEP2011.pdf

FED, Board of Governors of the Federal Reserve System. (2020). Federal Reserve announces extensive new measures to support the economy. Recuperado de: https://www.federalreserve.gov/newsevents/pressreleases/monetary20200323b.htm 
Fondo Monetario Internacional (FMI). (2018). Politica monetaria y actividad de los bancos centrales. Recuperado de: https://www.imf.org/ es/About/Factsheets/Sheets/2016/08/01/16/20/Monetary-Policy-and-Central-Banking

FUNDEU BBVA. (2015). Recomendaciones: preanuncio (monetario), mejor que forward guidance. Recuperado de: https://www.fundeu. es/recomendacion/preanuncio-monetario-forward-guidance/

Gertler, M. \& Karadi, P. (2011). A model of unconventional monetary policy. Journal of Monetary Policy, 58.

Gil, J. (2015). Postura de política monetaria ante fluctuaciones de la economía: una revisión de la evolución teórica. Finanzas y Políticas Económicas, 7(2, julio-diciembre), 381-440.

Jiménez, M. (2019). Causas de quiebra de bancos en el 2003. Hoy Digital. Recuperado de: http://hoy.com.do/citan-causas-quiebrabancos-2003-2/

Kang, K. \& Syd, M. (2008). El camino a la recuperación: las lecciones de Japón. Los problemas de liquidez e insolvencia requieren una estrategia. Finanza \& Desarrollo.

Kensuke, K. (2011). ¿Qué ha aprendido Japón de la crisis financiera?, NIPPON. Recuperado de: https://www.nippon.com/es/currents/ d00360/

Keynes, J. M. (1936). The General Theory of Employment, Interest, and Money. Nueva York, Harcourt, Brace.

Krugman, P. (1998). It's Baack: Japan's Slump and the Return of the Liquidity Trap. Brookings paper on economic activity.

Léco en Bref. (2019). Les politiques monétaires non conventionnelles. Recuperado de: https://abc-economie.banque-france.fr/sites/default/files/ medias/documents/l-eco-en-bref-politiques-monetaires-non-conventionnelles-2019-02-21_0.pdf

La Finance Pour Tous (2015). Les politiques monétaires non conventionelle. Recuperado de: https://url2.cl/wZCzy

Laborde, A. (2020). La Reserva Federal mantendrá los tipos próximos a cero hasta que la economía se recupere. El Pais. Recuperado de: https://elpais.com/economia/2020-04-29/la-reserva-federal-deee-uu-mantiene-los-tipos-de-interes-proximos-a-cero.html 
Ley 183-02, del 21 de noviembre, que aprueba la ley Monetaria y Financiera. Gaceta Oficial, 3 de diciembre de 2002, Núm. 10187.

Mankiw, G. (2010). Macroeconomics. Harvard University, 7th ed. ISBN13: 978-1-4292-1887-0. Recuperado de: http://irfanlal.yolasite. com/resources/N.\%20Gregory\%20Mankiw\%20Macroeconomics\%2C\%207th\%20Edition\%20\%20\%20\%202009.pdf

Milliken, D. (2020). Bank of England to finance UK government if markets turn sour. REUTERS, Recuperado de: https://www.reuters. com/article/us-health-coronavirus-britain-boe/bank-of-england-tofinance-uk-government-if-markets-turn-sour-idUSKCN21R0PA

Montas, J. (s.f). La crisis bancaria y sus causas: una reflexión sobre la crisis bancaria dominicana. Recuperado de: http://economia.gob.do/ mepyd/wp-content/uploads/archivos/despacho/publicaciones/ crisis-bancarias-sus-lecciones.pdf

Ping, S. (2020). Coronavirus: Government agrees emergency funding deal with Bank. BBC News. Recuperado de: https://www.bbc. com/news/business-52226482

Prats, E. \& Victoria, O. (2012). Derecho de la Regulación Monetaria y Financiera. (2a. ed.). Santo Domingo. IUS NOVUM. ISBN 9789945864861.

Princeton University Press. Liquidity Trap. Recuperado de: http://assets. press.princeton.edu/chapters/reinert/6article_ito_liquidity.pdf

Reglamento sobre el programa monetario e instrumentos de política monetaria. Santo Domingo, R. D., 23 de noviembre 2002. (Derogado)

Reserve Bank of Australia (RBA). (2020). Supporting the Economy and Financial System in Response to COVID-19. Recuperado de: https:// www.rba.gov.au/covid-19/

Samuelson, P. \& D. Nordhaus, W. (2015). Macroeconomia. Con aplicaciones para Latinoamérica. (19na ed.). México: McGRAW-HILL Interamericana Editores, SA. ISBN 978-970-10-5499-4. Recuperado de: http://cisprocr.com/cispro/system/files/Macroeconomia\% 20Samuelson.pdf

Sexton, R. \& Fortuna, P. (2005). Exploring Economics. (3ra ed.). Canadá: Nelson College Indigenous. ISBN 0-17-641482-7.

Suprema Corte de Justicia, 4 de agosto de 2004. B.J. 1125.91 
Tomás, F. (1994). “De la arbitrariedad de la administración”. Madrid: Civitas.

Trichet, J. (2010): «State of the Union: the financial crisis and the ECB's response between 2007 and 2009», Journal of Common Market Studies, 48, págs. 7-19.

United State of America. The Federal Reserve Act, entacted in december 23, 1913. Chapter 6 of the 62nd Congress, As Amended Through P.L. 115-174, 12 U.S.C. 221, art. 13 Sec. 2(A). Recuperado de: https:// www.govinfo.gov/app/search/\%7B\%22query\%22\%3A\%22federal\%20reserve\%20act $\% 22 \% 2 \mathrm{C} \% 22$ offset $\% 22 \% 3 \mathrm{~A} 0 \% 7 \mathrm{D}$

Vérgez, J. (2015). Aspectos jurídicos e institucionales de las politicas monetarias de los principales Bancos Centrales ante la actual crisis financiera. Madrid: DYKINSON, S.R.L. ISBN: 978-84-9085-430-3. Recuperado de: https://books.google.com.do/books?id=sGW7CwAAQBAJ\&pg=PA1 26\&dq= politicas + monetarias + no + convenc ionales \&hl=es\&sa =X\&ved=0ahUKEwiamrv0z4bhAhXxqlkKHScHCnEQ6AEIOjAD\#v=onepage $\& \mathrm{q}=$ politicas $\% 20$ monetarias $\%$ 20no\%20convencionales\&f=false, pág. 136. 